\title{
A Framework of Graduate Employment Recommendation System and Key Technologies
}

\author{
Shengbo Shi, a , Hexin Lv ${ }^{1, b}$ \\ ${ }^{1}$ School of Information Science and Technology, Zhejiang Shuren University, Hangzhou, 310015, \\ China \\ aemail: workshirley@yeah.net, bemail:hexin1024@sina.com
}

Keywords: Graduated Employment; Recommendation System; Recommendation Model;

\begin{abstract}
More and more websites have realized the importance of providing personalized recommendation by Recommendation System. As a supplement and improvement of Search Engine System, Recommendation System can be applied in the graduate employment websites to supply personalized employment guidance and recommendation to each graduate and improve the graduate employment service in China. In this paper, the main idea was to construct the framework and discuss the key technologies of Graduate Employment Recommendation System. Firstly, the graduate characteristic model, the recommendation model, and the recommendation rank and output model were discussed to construct the framework. Then, the key technologies such as graduate characteristic technologies, similarity algorithms, and neighbor selection mechanism were discussed to achieve the Graduate Employment Recommendation System. The experimental simulation result has shown that the recommendation system can be applied in graduate employment website and has a good performance.
\end{abstract}

\section{Introduction}

Since the enrollment expansion of Chinese colleges and universities, the number of college graduates has been increasing year by year [1]. However, the current graduate employment service in Chinese colleges is not enough to provide accurate and effective personalized employment guidance and recommendation for each graduate. In the initial stage for occupation, many graduates lack of target and pertinence and spend a lot of time and effort to apply for the jobs which are not suitable for them, which leads to the more and more difficult employment and improve the cost for college graduate and society.

More and more consideration of providing personalized recommendation, especially, it is necessary to adopt the new technologies and methods to construct the Graduate Employment Recommendation Systems to perfect the Chinese college graduate employment service and improve the quality and efficiency.

In this paper, we firstly introduce the related work of Recommendation System, especially the recommendation algorithms and recommendation methods in the following section. Section 3 presents a framework of Graduate Employment Recommendation Systems based on improved collaborative filtering algorithm. Section 4 discusses the key technologies for Graduate Employment Recommendation Systems such as the graduate characteristic extraction, the similarity algorithm and the Neighbors Selection mechanism. Section 5 presents the experimental results and analysis, and section 6 concludes the paper.

\section{Related Work}

Chen et al, [2] combined with the recommended results and collaborative filtering to recommend the interested goods for targeted users. Xue et al, [3] proposed a clustering method, which divides the users into k class and chooses the all of users in the nearest class as the neighbors. Tomoharu et al, [4] presented the maximum entropy principle collaborative filtering. Park et al, [5] combined collaborative filtering with search tool to enhance the Yahoo!. Sarwar et al, [6] applied the singular 
value decomposition algorithm to the recommender system. After KDDCup [7] and Netflix [8], there are hundreds of algorithms for the integration and improving the Recommendation System. Salakhutdinov et al, [9,10] proposed a Probability Matrix Factorization (PMF) algorithm and Bayesian probabilistic matrix factorization (BPMF) algorithm, which can effectively deal with large data. BPMF uses Markov Montecalo algorithm for parameter estimation, which has a certain improvement compared with PMF. Wang et al, [11] summarized the context aware personalized recommendation method. Yang et al, [12] proposed the real-time evolution of collaborative filtering algorithm, giving a higher weight of the new score to update the similarity of User and Item. In 2011, Jacob Abernethy in UC Berkeley et al [13], proposed OCF-SGD algorithm to achieve real-time collaborative filtering. Huang [14] improves the recommendation speed by introducing the clustering technique and the improved real-time collaborative filtering algorithm, and realizes the personalized recommendation in real time.

Recommendation System can be realized by two approaches [15, 16, 17]: one is the social approach: Collaborative Filtering Recommendation [18] is a kind of method which can be used to approximate the personalization; the other is a personalized approach, Content-based Recommendation [19] is a kind of method which is directly to find the user's specific preferences. Both of the two approaches, there are many specific methods to achieve the recommendation, such as machine learning, deep learning, complex networks and data mining, etc.

\section{A Framework of Graduate Employment Recommendation Systems}

With the continuous promotion of graduate employment information, the traditional “Recommendation Form for Graduates' Job Application”, a written material, can' t meet the needs of personalized employment recommendation. The employment websites for college graduate mainly supply information search engine to help graduates to filter the information, which is always incomplete. Thus, the Recommendation System should be introduced to the graduate employment websites to supply decision support service for the graduates and help them to carry out career planning. A framework of Graduate Employment Recommendation System is discussed in this section.

\section{$>\quad$ Graduate Characteristic Model}

Recommendation System is always be realized through 3stages: the construction of User Characteristic Model, the implementation of Recommendation Algorithm, the Output of the Recommendation Result. Among them, the key is the construction of the User Characteristic Model. The construction of User Characteristic Model is a process of acquiring and maintaining the knowledge related to user interest, demand or habits, and the result of the process is to build a User Characteristic Model that represents the user' specific background knowledge, interest or needs.In Graduate Employment Recommendation System, the specific user is the Graduate, thus we should extract the graduate employment characteristics, such as professional knowledge, professional skills, employment preference, employment behavior, employment psychology and social characteristic, etc.

\section{$>$ Recommendation Model}

As the mainstream recommendation approaches, the Content-based Recommendation and the Collaborative Filtering Recommendation are widely applied. Collaborative Filtering makes recommendations for target user by finding a set of neighbors, whose preference are similar with the target user and then recommending the neighbors' preferred items to the target user. The advantages of Collaborative Filtering are: the recommended items should not be considered, the serendipitous recommendations can be made, and less interference to the target user. In Graduate Employment Recommendation System, a set of similar graduates (as the neighbors) and their preferred job can be found and make recommendations to the target graduate.

\section{$>$ Recommendation Rank and Output Model}

Through the Recommendation Model, we have obtained a set of neighbor graduates and their preferred jobs. Now, the problem is how to rank these preferred jobs for the target graduate to 
improve the recommendation effect? The solution is taking the preference as the vector to calculate the similarity between graduates to find the neighbors, and then predict the recommended jobs and make ranked list for the target graduate according to the weight of the similarity and the preference of neighbors.

$>$ Recommendation System Framework

Combining with the above models, we constructed the following Recommendation System Framework as Fig.1.

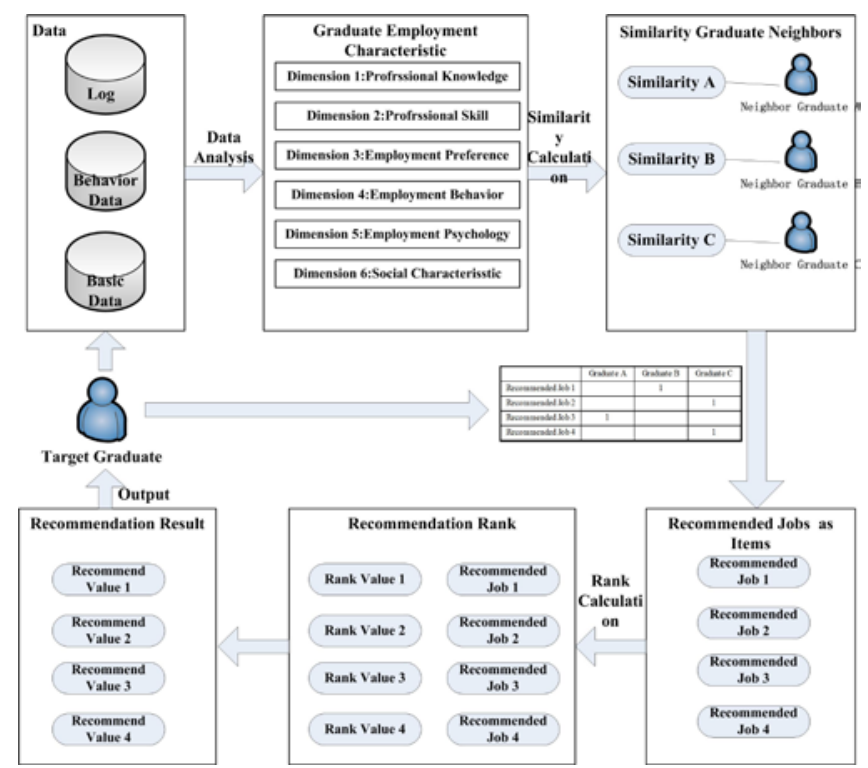

Fig.1: Recommendation System Framework

In the above framework of Graduate Employment Recommendation System, the first step is to analyst the graduate employment characteristic through the log, behavior and basic database. The second step is to calculate the similarity between graduates and find out the neighbor graduates. The third step is to recommend the neighbors' preferred jobs as the pre-recommendation items. The forth step is to rank the pre-recommendation items according to the similarity and rank calculation. The final step is to output the ranked recommendation items to target graduate.

\section{Key technologies of Graduate Employment Recommendation Systems}

\section{$>$ Graduate Characteristic}

The basis of Graduate Employment Recommendation System is how to extract the graduate' $\mathrm{s}$ employment preference. Graduates have many ways to supply their explicit data and implicit data to the websites. Examples of the association of the data and characteristic are shown as Table 1.

Table 1: Extraction of Graduate Employment Characteristic

\begin{tabular}{|c|c|c|}
\hline Graduate Employment Data & Type & $\begin{array}{c}\text { Extracted Graduate Employment } \\
\text { Characteristic }\end{array}$ \\
\hline $\begin{array}{l}\text { Basic Data ( e.g. the data from Register Information, Resume } \\
\text { and Career Testing should be extracted by text analysis) }\end{array}$ & Explicit & $\begin{array}{l}\text { Professional Knowledge } \\
\text { Professional Skill } \\
\text { Employment Psychology } \\
\text { Social Relationship }\end{array}$ \\
\hline $\begin{array}{l}\text { Log and Behavior Data (e.g. } \\
\text { Collection Added) }\end{array}$ & $\begin{array}{l}\text { Explicit (Boolean, } \\
\text { the Value is } 0 \text { or } 1)\end{array}$ & $\begin{array}{l}\text { Employment preference } \\
\text { Employment Behavior }\end{array}$ \\
\hline $\begin{array}{l}\text { Log and Behavior Data (e.g. } \\
\text { the data from Tag Labels should be extracted by word analysis) }\end{array}$ & Explicit & $\begin{array}{l}\text { Employment preference } \\
\text { Employment Behavior }\end{array}$ \\
\hline $\begin{array}{l}\text { Log and Behavior Data (e.g. } \\
\text { the data from Click should be extracted by analysis ) }\end{array}$ & Implicit & $\begin{array}{l}\text { Employment preference } \\
\text { Employment Behavior }\end{array}$ \\
\hline $\begin{array}{l}\text { Log and Behavior Data (e.g. the data from Page Dwell Time } \\
\text { should be extracted by de-noising analysis) }\end{array}$ & Implicit & $\begin{array}{l}\text { Employment preference } \\
\text { Employment Behavior }\end{array}$ \\
\hline Log and Behavior Data (e.g. Submit Resume) & $\begin{array}{l}\text { Implicit (Boolean, } \\
\text { the Value is } 0 \text { or } 1 \text { ) }\end{array}$ & $\begin{array}{l}\text { Employment preference } \\
\text { Employment Behavior }\end{array}$ \\
\hline
\end{tabular}


The collection data should be weighted according to the graduate' s preference. Generally, the weight of explicit data is greater than implicit data. Also the data should be pre-processed by de-noising and normalization. After the pre-processing and weighed- processing, we can obtain a two-dimensional matrix of graduate preference as Fig.2. One dimensional is graduate list, the other dimensional is the job list, and the value is $[0,1]$.

$$
\mathrm{R}=\left(\begin{array}{ccc}
r_{11} & \cdots & r_{1 \mathrm{n}} \\
\vdots & \ddots & \vdots \\
r_{m 1} & \cdots & r_{m n}
\end{array}\right)
$$

Fig.2: Graduate Preference Matrix

In Fig.2, represents the job ' s score from graduate, and is set to be a number between 0 1, which means the graduate's preference for the job.

$>$ Recommendation Algorithm

After obtaining the Graduate Preference Matrix, we can calculate the similar graduate through similarity algorithm.

\section{a. Similarity Algorithm}

The mainstream of Similarity Algorithm includes Pearson Correlation Similarity, Euclidean Distance Similarity, Tanimoto Coefficient Similarity, and Cosine Similarity. In Graduate Employment Recommendation System, we take $\left(r_{m 1}, r_{m 2}, \ldots m r_{m n}\right)$ as a vector to calculate the similarity between graduates by Cosine Similarity algorithm. Cosine Similarity distinguishes the difference by direction, which is not sensitive to the absolute value and suitable to calculate the similarity of graduate's preference. Setting $R_{1}=\left(r_{11}, r_{12}, \ldots \ldots, r_{1 n}\right)$ and $R_{2}=\left(r_{22}, r_{22}, \ldots \ldots, r_{2 n}\right)$, the cosine similarity between $R_{1}$ and $R_{2}$ is:

$$
\cos \theta=\frac{\sum_{\mathrm{i}=1}^{\mathrm{n}}\left(r_{1 \mathrm{i}} \times r_{2 i}\right)}{\sqrt{\sum_{\mathrm{i}=1}^{\mathrm{n}}\left(r_{1 i}\right)^{2}} \times \sqrt{\sum_{\mathrm{i}=1}^{\mathrm{n}}\left(r_{2 i}\right)^{2}}}=\frac{R_{1}-R_{2}}{\left|R_{1}\right| \times\left|R_{2}\right|}
$$

\section{b.Similarity Neighbors Selection}

Fig.3: Cosine Similarity Between ${ }^{R_{1}}$ and ${ }^{R_{2}}$

After calculating the similarity, we need to choose the neighbors of the target graduate. The approaches of neighbor selection can be divided into two categories: one is Fix-size Neighborhoods; the other is Threshold-based Neighborhoods. The number of neighbors is limited by a-fixed similarity value by Threshold-based Neighborhoods and more advantage than Fix-size Neighborhoods.

\section{Results}

According to the above framework and key technology research, we import the random experimental data to carry out the relevant tests. According to the previous research, we set the corresponding weight of the graduate employment characteristic as shown in Table 2.

Table 2: Weight of the Graduate Employment Characteristic

\begin{tabular}{|c|c|c|}
\hline Specific Graduate Employment Characteristic & Weight & Graduate Employment Characteristic Category \\
\hline Major (from Resume) & 0.2 & Professional Knowledge \\
\hline Education (from Resume) & 0.1 & Professional Knowledge \\
\hline Qualification (from Resume) & 0.2 & $\begin{array}{c}\text { Employment Psychology } \\
\text { Employment preference }\end{array}$ \\
\hline Career Testing Result & 0.2 & Social Relationship \\
\hline Contacts circle & 0.1 & $\begin{array}{c}\text { Employment preference } \\
\text { Employment Behavior }\end{array}$ \\
\hline Collection Added & 0.03 & $\begin{array}{c}\text { Employment preference } \\
\text { Employment Behavior }\end{array}$ \\
\hline Tag Labels & 0.03 & $\begin{array}{c}\text { Employment preference } \\
\text { Employment Behavior }\end{array}$ \\
\hline Click & 0.03 & $\begin{array}{c}\text { Employment preference } \\
\text { Employment Behavior }\end{array}$ \\
\hline Page Dwell Time & 0.05 & $\begin{array}{c}\text { Employment preference } \\
\text { Employment Behavior }\end{array}$ \\
\hline Submit Resume & 0.06 & \\
\hline
\end{tabular}


Based on the weight of the graduate employment characteristic and the graduate employment data, the graduate employment preference matrix is shown as Table 3.

Table 3: Graduate Employment Preference Matrix

\begin{tabular}{|c|c|c|c|c|c|c|c|c|c|c|}
\hline & Job1 & Job2 & Job3 & Job4 & Job5 & Job6 & Job7 & Job8 & Job9 & Job10 \\
\hline Graduate A & 0.56 & 0 & 0.94 & 0 & 0 & 0 & 0.36 & 0.87 & 0 & 0 \\
\hline Graduate B & 0 & 0 & 0 & 0.53 & 0 & 0.94 & 0 & 0 & 0 & 0 \\
\hline Graduate C & 0 & 0 & 0.63 & 0 & 0 & 0 & 0.94 & 0 & 0 & 0.89 \\
\hline Graduate D & 0 & 0.97 & 0 & 0 & 0 & 0.84 & 0 & 0.46 & 0 & 0 \\
\hline Graduate E & 0.4 & 0.81 & 0 & 0 & 0.63 & 0 & 0 & 0 & 1 & 0 \\
\hline Graduate F & 0 & 0.43 & 0 & 0 & 0.94 & 0 & 0 & 0.97 & 0 & 0 \\
\hline Graduate G & 0.97 & 0 & 0.3 & 0 & 0 & 0 & 0 & 0.3 & 0 & 0 \\
\hline Graduate H & 0.86 & 0 & 0.43 & 0 & 0 & 0.97 & 0 & 0.3 & 0 & 0 \\
\hline Graduate I & 0.3 & 1 & 0.6 & 0 & 0 & 0 & 0 & 0 & 0.81 & 0 \\
\hline Graduate J & 0 & 0 & 0.97 & 0 & 0 & 0 & 0.81 & 0 & 0.3 & 0 \\
\hline
\end{tabular}

According to the graduate employment preference matrix and similarity algorithm, the similarity between the graduates is calculated, as shown in Table 4.

Table 4: Similarity between the Graduates

\begin{tabular}{|c|c|c|c|c|c|c|c|c|c|c|c|}
\hline & Job1 & Job2 & Job3 & Job4 & Job5 & Job6 & Job7 & Job8 & Job9 & Job10 & Similarity with Graduate A \\
\hline Graduate A & 0.56 & 0 & 0.94 & 0 & 0 & 0 & 0.36 & 0.87 & 0 & 0 & 1 \\
\hline Graduate B & 0 & 0 & 0 & 0.53 & 0 & 0.94 & 0 & 0 & 0 & 0 & 0 \\
\hline Graduate C & 0 & 0 & 0.63 & 0 & 0 & 0 & 0.94 & 0 & 0 & 0.89 & 0.447803729 \\
\hline Graduate D & 0 & 0.97 & 0 & 0 & 0 & 0.84 & 0 & 0.46 & 0 & 0 & 0.203387823 \\
\hline Graduate E & 0.4 & 0.81 & 0 & 0 & 0.63 & 0 & 0 & 0 & 1 & 0 & 0.11046451 \\
\hline Graduate F & 0 & 0.43 & 0 & 0 & 0.94 & 0 & 0 & 0.97 & 0 & 0 & 0.41242045 \\
\hline Graduate G & 0.97 & 0 & 0.3 & 0 & 0 & 0 & 0 & 0.3 & 0 & 0 & 0.123668979 \\
\hline Graduate H & 0.86 & 0 & 0.43 & 0 & 0 & 0.97 & 0 & 0.3 & 0 & 0 & 0.774615966 \\
\hline Graduate I & 0.3 & 1 & 0.6 & 0 & 0 & 0 & 0 & 0 & 0.81 & 0 & 0.349425014 \\
\hline Graduate J & 0 & 0 & 0.97 & 0 & 0 & 0 & 0.81 & 0 & 0.3 & 0 & 0.641851957 \\
\hline
\end{tabular}

As shown as the above table 3 , the similarity with Graduate A (target graduate) is ranked: Graduate $\mathrm{H}>$ Graduate $\mathrm{J}>$ Graduate $\mathrm{C}>$ Graduate $\mathrm{F}>$ Graduate $\mathrm{I}>$ Graduate D $>$ Graduate $\mathrm{G}>$ Graduate E $>$ Graduate B. If we choose 0.5 as the threshold, the recommended jobs are Job 6 and Job 9.

\section{Conclusions}

In this paper, we proposed a framework of Graduate Employment Recommendation System, discussed the key technologies, and demonstrated the application of the proposed framework and technologies. We first constructed Graduate Characteristic Model, Recommendation Model, and Recommendation Rank and Output Model. Then we built a framework of Graduate Employment Recommendation System based on the above models. We also discussed Graduate Characteristic Technology and Recommendation Algorithm to achieve the System. Finally, we evaluated the system performance of the proposed framework and technologies. For future work, we are considering the development of recommendation algorithm.

\section{Acknowledgement}

In this paper, the research was sponsored by Ministry of Education, Humanities and Social Science Projects of China (No.12YJA880085), and the Foundation of Zhejiang Educational 
Committee (No.Y201534557).

\section{References}

[1] Lv Hexin, Zhang Qing, Song Bin. Constructing Employment Management Model for College Graduates [J]. Research in Higher Education of Engineering, 2014(06):118-122. (in Chinese).

[2] Chen M C, Chen L S, Hsu F H, et al. HPRS: A profitability based recommender system[C]. Industrial Engineering and Engineering Management, 2007 IEEE International Conference on. IEEE, 2007: 219-223.

[3] Xue G R, Lin C, Yang Q, et al. Scalable collaborative filtering using cluster-based smoothing[C]. Proceedings of the 28th annual international ACM SIGIR conference on Research and development in information retrieval, ACM, 2005: 114-121.

[4] Iwata T, Saito K, Yamada T. Modeling user behavior in recommender systems based on maximum entropy[C]. Proceedings of the 16th international conference on World Wide Web, ACM, 2007: 1281-1282.

[5] Park S T, Pennock D M. Applying collaborative filtering techniques to movie search for better ranking and browsing[C]. Proceedings of the 13th ACM SIGKDD international conference on Knowledge discovery and data mining, ACM, 2007: 550-559.

[6]Sarwar B, Karypis G, Konstan J, et al. Application of dimensionality reduction in recommender system-a case study[R].Proc. ACM WebKDD 2000 Web Mining for E-Commerce Workshop, 2000.

[7]Edward F. Harrington. Online Ranking/Collaborative filtering using the Perceptron Algorithm [C]. Proceedings of the Twentieth International Conference on Machine Learning, 2003:176-184.

[8]S. Funk. Netflix update: Try this at home. http://sifter.org/ simon/journal/20061211.html.

[9]Salakhutdinov R, Mnih A. Probabilistic Matrix Factorization[C]. In: NIPS, 2007, 1(1): 2.1.

[10] Salakhutdinov R, Mnih A. Bayesian probabilistic matrix factorization using Markov chain Monte Carlo[C]. In: Proceedings of the 25th international conference on Machine learning. ACM, 2008: 880-887.

[11] WANG Li-Cai, MENG Xiang-Wu, ZHANG Yu-Jie. Context-Aware Recommender Systems [J]. Journal of Software, 2012, 23(1):1-20. ( in Chinese).

[12]Q Yang, X Xue. Transfer learning for collaborative filtering via a rating-matrix generative model [C]. ICML '09 Proceedings of the 26th Annual International Conference on Machine Learning, 2009, 617-624.

[13] Jacob Abernethy, Kevin Canini, John Langford, Alex Simma. Online Collaborative Filtering [J]. Di.ens.fr, 2011:271-280.

[14] C Huang.Cluster ensembles in collaborative filtering recommendation [J]. Applied Soft Computing, Volume 12 Issue 4, April, 2012:1417-1425.

[15] Xu H L, Wu X, Li X D,et al. Comparison Study of Internet Recommendation System [J]. Journal of Software, 2009, 20 (2): 350-362 (in Chinese).

[16] Min Sung-hwan, Han Ingoo. Detect ion of the Customer Timevariant Pattern for Improving Recommender Systems[J] .Expert Systems with Applications, 2005, 28 (2):189-199.

[17] Resnick P, Varian H R. Recommender Systems. Communications of the ACM, 1997,40( 3) : 56-58.

[18] LENG Ya-Jun, LU Qing, LIANG Chang-Yong. Survey of Recommendation Based on Collaborative Filtering[J]. Pattern Recognition and Artificial Intelligence,2014(08):720-731.

[19] Balabanovic M , S hoham Y .Content-based, collaborative recommendation [J]. Communications of the ACM , 1997 , 40(3):66-72. 